\title{
ON COMPACTIFICATIONS OF INFINITE-DIMENSIONAL SPACES
}

\author{
VITALIJ A. CHATYRKO
}

\begin{abstract}
For every separable metrizable space $X$ with trind $X \neq \infty$ there exists a countable ordinal number $\beta(X) \geq$ trind $X$ such that for every countable ordinal number $\gamma \geq \beta(X)$ there exists a compactification $c_{\gamma} X$ of the space $X$ with trind $c_{\gamma} X=\gamma(\beta(X)=\operatorname{trind} X$, if $\operatorname{trInd} X \neq \infty)$.
\end{abstract}

\section{Introduction}

Throughout this note we shall consider only separable metrizable spaces. The necessary information about notions and notations we use can be found in [AP], [E].

It is well known the following Hurewicz's result (see for example [AP]):

(*) for every space $X$ with ind $X=n$ there exists a compactification $c X$ of the space $X$ with ind $c X=n, n=0,1,2, \ldots$

It is known ( see [E]) that

(**) for every space $X$ with trind $X \neq \infty$ there exists a compactification $c X$ with trind $c X \neq \infty$ (trind is the transfinite extension of the dimension function ind ).

However the exact extension of proposition $(*)$ to the transfinite case is impossible. In [Lu1] Luxemburg has proved that for any limit ordinal number $\alpha: \omega \leq \alpha<\omega_{1}$ there exists a complete strongly countable-dimensional space $X_{\alpha}$ with trind $X_{\alpha}=\alpha$ such that for every compactification $c X_{\alpha}$ of the space $X_{\alpha}$ we have trind $c X_{\alpha}>$ trind $X_{\alpha}$ (by definition we assume $\infty>\alpha$ for every ordinal number $\alpha$ ).

Recall that trind $Z \leq \operatorname{trind} Y$, if $Z \subseteq Y$.

In [E] Engelking has remarked the following open

Problem. Evaluate the increase of trind in the process of compactifying a separable metrizable space.

Received November 11, 1996. 
One of the results of this paper is

THEOREM 1. For every space $X$ with trind $X \neq \infty$ there exists a countable ordinal number $\beta(X) \geq$ trind $X$ such that for every countable ordinal number $\gamma \geq \beta(X)$ there exists a compactification $c_{\gamma} X$ of the space $X$ with trind $c_{\gamma} X=\gamma$. Moreover, if $\operatorname{trInd} X \neq \infty$, then $\beta(X)=\operatorname{trind} X(\operatorname{tr} \operatorname{Ind} i$ is the transfinite extension of the dimension function $\mathrm{Ind})$.

Note that for every space $X$ with trind $X \neq \infty$ we have trind $X<\omega_{1}$ [AP].

\section{The case of the locally compact noncompact spaces}

Let $X, Y$ be topological spaces. The notation $X \simeq Y$ will mean that the spaces $X$ and $Y$ are homeomorphic and the notation $X \hookrightarrow Y$ will mean that the space $X$ is homeomorphic to a subset of the space $Y$. Let $X \subset Y$. The notation $[X]_{Y}$ will mean the closure of the space $X$ in the space $Y$.

We shall need the following Theorem 2 which is a corollary from a fact established by Aarts and van Emde Boas [AE]. For the sake of completeness, let us outline its proof.

Let $X$ be a locally compact noncompact space and $b X=X \cup\{p\}$ be the one-point compactification of the space $X$, where $p$ is the compactification point.

It is evident that there exists a continuous function $f: b X \longrightarrow I=[0,1]$ such that $f^{-1}\{0\}=p$. Put

$$
X_{f}=\{(x, f(x)): x \in X\} \subset X \times I, b X_{f}=\{(x, f(x)): x \in b X\} \subset b X \times I .
$$

Note that $X \simeq X_{f}$ and $b X \simeq b X_{f}$. Let $\operatorname{pr}_{I}: b X \times I \longrightarrow I$ be the projection of the compact space $b X \times I$ onto the closed interval $I$. It is easy to see that there exists a sequence $\left\{c_{n}\right\}_{n=1}^{\infty}$ of points from $X_{f}$ with $\lim _{n \rightarrow \infty}\left(c_{n}\right)=$ $\{p\} \times\{0\}$ such that $x_{n+1}<x_{n}$ for any $n \in \mathrm{N}$ and $\lim _{n \rightarrow \infty}\left(x_{n}\right)=0$, where $x_{n}=\operatorname{pr}_{I}\left(c_{n}\right)$.

Let $A$ be a nondegenerated AR-compactum and $S=\left\{u_{n}\right\}_{n=1}^{\infty}$ be a countable everywhere dense subset of $A$. Define the mapping $h:\left\{x_{n}\right\}_{n=1}^{\infty} \longrightarrow A$ as follows: $h\left(x_{n}\right)=u_{n}$ for any $n \in \mathrm{N}$. Let $g:(0,1] \longrightarrow A$ be a continuous extension of the mapping $h$. Put

$$
W=b X \times I \times A, Z=\{(x, f(x), g(f(x))): x \in X\} \subset X \times(0,1] \times A \subset W
$$

It is evident that $X \simeq Z,[Z]_{W}=Z \cup(\{p\} \times\{0\} \times A)$ and $[Z]_{W} \backslash Z \simeq A$. Denote $[Z]_{W}=K[X, A]$.

We have proved

TheORem 2. Let $X$ be a locally compact noncompact space and $A$ be a non- 
degenerated AR-compactum. Then there exists a compactification $c X$ of the space $X$ such that $c X \backslash X \simeq A$.

We will say here that a dimension function $F$ is monotone, if for every space $X$ and any its closed subset $Y$ we have $F Y \leq F X$.

We will say that a dimension function $F$ is $\omega_{1}$-bounded, if for any space $X$ we have $F X<\omega_{1}$ or $F X=\infty$.

Let $X$ be a compact space and $Y$ be a closed subset in $X$ with $F Y \neq \infty(F Y=\beta)$, where $F$ is a dimension function. Moreover, let for every closed subset $Q \subset X$ such that $Q \cap Y=\emptyset$, we have $F Q \neq \infty(F Q \leq \alpha$ and $\beta \geq \alpha)$. If $F X \neq \infty(F X \leq \alpha+\beta)$, then we will say that the dimension function $F$ has the (strong ) Dowker property.

Corollary 1. Let $F$ be a monotone $\omega_{1}$-bounded dimension function which has the Dowker property. Moreover, let $\sup \left\{F P^{\alpha}: \alpha<\omega_{1}\right\}=\omega_{1}$, where $P^{\alpha}, \alpha<\omega_{1}$, are AR-compacta. Then for every locally compact noncompact space $X$ such that $F Q \neq \infty$ for any compactum $Q \subset X$ we have $\sup \{F c X: c X$ is a compactification of space $X$ with $F c X \neq \infty\}=\omega_{1}$.

Recall $[\mathrm{KM}]$ that any ordinal number $\alpha$ can be uniquely represented as $\alpha=\omega^{\eta_{1}} \cdot n_{1}+\ldots+\omega^{\eta_{k}} \cdot n_{k}$, where $n_{i} \in \mathrm{N}$ and $\eta_{1}>\ldots>\eta_{k} \geq 0$ are ordinal numbers. Note that for every ordinal number $\beta \geq \omega^{\eta_{1}+1}$, we have $\alpha+\beta=\beta$.

COROllary 2. Let $F$ be a monotone dimension function which has the strong Dowker property. Moreover, let for every countable ordinal number $\gamma$ there exists an AR-compactum $A^{\gamma}$ with $F A^{\gamma}=\gamma$. Then for every locally compact noncompact space $X$ such that $F Q \leq \alpha$ for every compactum $Q \subset X$, and for any ordinal number $\gamma: \alpha \leq \gamma<\omega_{1}$ we have $\gamma \leq F\left(K\left[X, A^{\gamma}\right]\right) \leq \alpha+\gamma$. In particular, if $\alpha=\omega^{\eta_{1}} \cdot n_{1}+\ldots+\omega^{\eta_{k}} \cdot n_{k}$, where $n_{i} \in \mathrm{N}$ and $\eta_{1}>\ldots>\eta_{k} \geq 0$ are ordinal numbers, then for every countable ordinal number $\beta \geq \omega^{\eta_{1}+1}$, we have $F\left(K\left[X, A^{\beta}\right]\right)=\beta$.

Recall [E] the definitions of dimension functions trind, trInd, D, trdim which are different transfinite extensions of the finite dimension dim in the class of separable metrizable spaces.

Let $X$ be a space. Define

(i) trind $X=-1 \Leftrightarrow X=\emptyset$;

(ii) trind $X \leq \alpha$, where $\alpha$ is an ordinal number, if for every point $x \in X$ and each neighborhood $V$ of the point $x$ there exists an open set $U \subset X$ such that $x \in U \subset V$ and trind $\operatorname{Fr} U<\alpha$;

(iii) $\operatorname{trind} X=\alpha$ if trind $X \leq \alpha$ and the inequality trind $X \leq \beta$ holds for no $\beta<\alpha$;

(iv) trind $X=\infty$ if trind $X \leq \alpha$ holds for no ordinal number $\alpha$. 
The definition of trInd one can get through the substitution of the point $x$ in (ii) from the definition above with a closed subset of the space $X$.

Observe that for each ordinal number $\alpha$ there exist a uniquely determined limit number $\lambda(\alpha) \geq 0$ and an integer $n(\alpha) \geq 0$ such that $\alpha=\lambda(\alpha)+n(\alpha)$.

We let $\mathrm{D}(\emptyset)=-1$, and for every non-empty space $X$ we define $\mathrm{D}(X)$ as the smallest ordinal number $\alpha$ such that there exists a closed cover $\left\{A_{\beta}\right\}_{\beta \leq \lambda(\alpha)}$ of the space $X$ satisfying the following conditions:

(D1) The union $\cup\left\{A_{\beta}: \delta \leq \beta \leq \lambda(\alpha)\right\}$ is closed for every $\delta \leq \lambda(\alpha)$;

(D2) For every $x \in X$ the set $\left\{\beta \leq \lambda(\alpha): x \in A_{\beta}\right\}$ has a largest element;

(D3) $\operatorname{dim} A_{\beta}<\infty$ for every $\beta<\lambda(\alpha)$, and $\operatorname{dim} A_{\lambda(\alpha)} \leq n(\alpha)$;

if no such ordinal number exists, we let $\mathrm{D}(X)=\infty$.

It is clear that $\mathrm{D} Z \leq \mathrm{D} Y$, if $Z \subseteq Y$.

Let $L$ be an arbitrarary set. By Fin $L$ we shall denote the collection of all finite, non-empty subsets of $L$. Let $M$ be a subset of Fin $L$. For $\sigma \in\{\emptyset\} \cup \operatorname{Fin} L \quad$ we put $M^{\sigma}=\{\tau \in \operatorname{Fin} L \mid \quad \sigma \cup \tau \in M$ and $\sigma \cap \tau=\emptyset\}$. Let $M^{a}=M^{\{a\}}$.

Define the ordinal number $\operatorname{Ord} M$ inductively as follows

(i) $\operatorname{Ord} M=0$ iff $M=\emptyset$,

(ii) $\operatorname{Ord} M \leq \alpha$ iff for every $a \in L \quad \operatorname{Ord} M^{a}<\alpha$,

(iii) $\operatorname{Ord} M=\alpha$ iff $\operatorname{Ord} M \leq \alpha \quad$ and $\operatorname{Ord} M<\alpha$ is not true, and

(iv) $\operatorname{Ord} M=\infty \quad$ iff $\operatorname{Ord} M>\alpha \quad$ for every ordinal number $\alpha$.

Let $X$ be a non-empty space. A finite sequence $\left\{\left(A_{i}, B_{i}\right)_{i=1}^{m}\right\}$ of pairs of disjoint closed sets in the space $X$ is called inessential if we can find open sets $O_{i}, i=1, \ldots, m$ such that $A_{i} \subset O_{i} \subset\left[O_{i}\right]_{X} \subset X \backslash B_{i} \quad$ and $\quad \bigcap_{i=1}^{m} \operatorname{Fr} O_{i}=\emptyset$. Otherwise it is called essential.

Put $L(X)=\{(A, B) \mid A, B \subset X, \quad$ closed, disjoint $\}$ and $M_{L(X)}=$ $\{\sigma \in \operatorname{Fin} L(X) \mid \sigma$ is essential in $X\}$.

We let $\operatorname{trdim}(\emptyset)=-1$, and for every non-empty space $X$ we define $\operatorname{trdim} X=\operatorname{Ord} M_{L(X)}$.

Note that the dimension functions trind, trInd, D, trdim are monotone, $\omega_{1}$-bounded and they have the strong Dowker property (for the dimensions trind, trInd about the strong Dowker property see for example [B1], for D [He1], for trdim - [Ha]).

In [He2] Henderson have constructed AR-compacta $H^{\alpha}, \alpha<\omega_{1}$, and have proved that $\operatorname{trInd} H^{\alpha}=\alpha, \alpha<\omega_{1}$. Observe that $F H^{\alpha}=\alpha, \alpha<\omega_{1}$, for $F=\mathrm{D}$ ( see [Ch]), trdim (see [B1]). Moreover, from Levshenko's inequality [Le] $\operatorname{trInd} X \leq \omega \cdot \operatorname{trind} X$, which is true for any compact space $X$, we have $\sup \left\{\operatorname{trind} H^{\alpha}: \alpha<\omega_{1}\right\}=\omega_{1}$.

Recall the construction of Henderson's AR-compacta $H^{\alpha}, \alpha<\omega_{1}[\mathrm{He} 2]$. Let $H^{1}=I=[0,1], p_{1}=\{0\} \in I$. Assume that for every $\beta<\alpha$ the compacta 
$H^{\beta}$ and the points $p_{\beta} \in H^{\beta}$ have already been defined. If $\alpha=\beta+1$, then we set $H^{\beta+1}=H^{\beta} \times I$ and $p_{\alpha}=\left(p_{\beta}, 0\right)$. If $\alpha$ is a limit ordinal number, then $K_{\beta}$ is the union of the $H^{\beta}$ and a half-open arc $A_{\beta}$ such that $A_{\beta} \cap H^{\beta}=\left\{p_{\beta}\right\}=\{$ endpoint of the arc $\left.A_{\beta}\right\}, \beta<\alpha$. Let us define $H^{\alpha}$ as the one-point compactification of the free sum $\bigoplus_{\beta<\alpha} K_{\beta}$ and let $p_{\alpha}$ be the compactification point.

Recall also [T] that trind $X \times I \leq \operatorname{trind} X+1$, for any space $X$.

Now it is easy to note that one can choose from the collection $\left\{H^{\alpha}: \alpha<\omega_{1}\right\}$ a new collection $\left\{P^{\alpha}: \alpha<\omega_{1}\right\}$ such that for every ordinal number $\alpha<\omega_{1}$ we have trind $P^{\alpha}=\alpha$.

REMARK 1. The dimension functions trind, trInd, D, trdim satisfy the conditions of Corollary 1, 2.

\section{The general case}

THEOREM 3. Let $X$ be a noncompact space and $c_{1} X$ be a compactification of the space $X$. Then for every nondegenerate AR-compact space $A$ and any point $p \in c_{1} X \backslash X$ there exists a compactification $c X$ of the space $X$ such that $c X \hookleftarrow c_{1} X \backslash\{p\}$ and $c X \backslash X \hookleftarrow A$.

Proof. Denote $X_{1}=c_{1} X \backslash\{p\} \hookleftarrow X$. Then $c X=K\left[X_{1}, A\right]$.

COROllary 3. Let $F$ be a monotone $\omega_{1}$-bounded dimension function which has the Dowker property. Moreover, let $\sup \left\{F P^{\alpha}: \alpha<\omega_{1}\right\}=\omega_{1}$, where $P^{\alpha}, \alpha<\omega_{1}$, are AR-compacta. Then for every noncompact space $X$ such that $X$ has a compactification $c_{1} X$ with $F c_{1} X \neq \infty$ we have $\sup \{F c X: c X$ is a compactification of space $X$ with $F c X \neq \infty\}=\omega_{1}$.

Corollary 4. Let $F$ be a monotone dimension function which has the strong Dowker property. Moreover, let for every countable ordinal number $\gamma$ there exists an AR-compactum $A^{\gamma}$ with $F A^{\gamma}=\gamma$. Then for every noncompact space $X$, such that $X$ has a compactification $c X$ with $F c X=\alpha$, and for any ordinal number $\gamma: \alpha \leq \gamma<\omega_{1}$ there exists a compactification $c_{\gamma} X$ with $\gamma \leq F c_{\gamma} X \leq \alpha+\gamma$. In particular, if $\alpha=\omega^{\eta_{1}} \cdot n_{1}+\ldots+\omega^{\eta_{k}} \cdot n_{k}$, where $n_{i} \in \mathrm{N}$ and $\eta_{1}>\ldots>\eta_{k} \geq 0$ are ordinal numbers, then for every countable ordinal number $\beta \geq \omega^{\eta_{1}+1}$ we have $F c_{\beta} X=\beta$.

REMARK 2. The dimension functions trind, trInd, D, trdim satisfy the conditions of Corollary 4.

For any space $X$ we will denote by $P(X)$ a closed subset of the space $X$ such that $X \backslash P(X)$ is the union of all finite-dimensional sets, open in $X$.

In [Lu1] Luxemburg has proved that 
(***) for any space $X$ with $\operatorname{trInd} X \neq \infty$ the set of all homeomorphisms $f: X \rightarrow I^{\omega}$ of the space $X$ to the Hilbert cube $I^{\omega}$ such that the equalities

(a) $F X=F\left([f X]_{I^{\omega}}\right)$,

(b) $P X=P\left([f X]_{I^{\omega}}\right)$,

where $F$ is one of the dimension functions trind, trInd, D, are satisfied contains an everywhere dense set of type $G_{\delta}$ in the space $C\left(X, I^{\omega}\right)$. In particular, there exists a compactification $c X$ with $F c X=F X$ and $P(c X)=P X$, where $F=$ trind, trInd or $\mathrm{D}$.

Kimura [Ki] has proved the same for trdim.

Let $F$ be one of the dimension functions trind, trInd, trdim or D. Recall (see for example [E] and [B1] for trdim) that if a space $X$ can be represented as the union of two closed subspaces $B_{1}$ and $B_{2}$ such that $F B_{i} \leq \alpha \geq \omega_{0}$ for $i=1,2$ and the subspace $B_{1} \cap B_{2}$ is finite-dimensional, then $F X \leq \alpha$.

THeOREM 4. Let $X$ be a noncompact space with $\operatorname{trInd} X \neq \infty$ and $F$ be one of the dimension functions trind, trInd, trdim or $\mathrm{D}$. Then for every countable ordinal number $\alpha \geq F X$ there exists a compactification $c_{\alpha, F} X$ such that $F c_{\alpha, F} X=\alpha$.

Proof. Let $\alpha$ be a countable ordinal number $\geq F X$ and let $c X$ be a compactification of the space $X$ such that $F c X=F X$ and $P(c X)=P X$ (see $\left({ }^{* *}\right)$ ). Consider a point $p \in c X \backslash X$. Observe that there exists an open finitedimensional set $U \subset c X$ such that $p \in U$. Let $A$ be an AR-compact with $F A=\alpha$. Set $c_{\alpha, F} X=K[c X \backslash\{p\}, A]$. Note that the compactification $c_{\alpha, F} X$ of the space $X$ can be represented as the union of two closed subspaces $B_{1}$ and $B_{2}$ such that $F B_{1} \leq F A=\alpha, F B_{2} \leq F X \leq \alpha$ and the subspace $B_{1} \cap B_{2}$ is finite-dimensional. Consequently $F c_{\alpha, F} X=\alpha$.

Theorem 1 follows proposition (**), Corollary 4 and Theorem 4. The same statements hold for dimensions trInd, D, trdim (see part 4.).

\section{Examples}

Let $L$ be the space of irrational numbers.

Observe that

(i) $L \times L \simeq L$;

(ii) $\bigoplus_{n=1}^{\infty} L_{n} \simeq L$, where $\bigoplus_{n=1}^{\infty} L_{n}$ is the free sum of the spaces $L_{n} \simeq L, n=1,2, \ldots$;

(iii) ind $L=0$.

Recall the construction of Smirnov's compacta $S^{\alpha}, \alpha<\omega_{1}$ [S]. Let $S^{0}$ be the one-point space. Assume that for every $\beta<\alpha$ the compacta $S^{\beta}$ have already been defined. If $\alpha=\beta+1$, then we set $S^{\beta+1}=S^{\beta} \times I$. If $\alpha$ is a limit 
ordinal number, then let us define $S^{\alpha}$ as the one-point compactification of the free sum $\bigoplus_{\beta<\alpha} S_{\beta}$, where $p_{\alpha}$ is the compactification point.

Note that

a) if $\left\{\alpha_{i}\right\}_{i=1}^{\infty}$ is a sequence of ordinal numbers such that $\alpha_{i}<\alpha_{i+1}$ and $\sup _{i} \alpha_{i}=\alpha<\omega_{1}$, then $S^{\alpha} \hookrightarrow\{b\} \cup \bigoplus_{i=1}^{\infty} S^{\alpha_{i}} \hookrightarrow S^{\alpha}$, where $\{b\} \cup \bigoplus_{i=1}^{\infty} S^{\alpha_{i}}$ is the one-point compactification of the free sum $\bigoplus_{i=1}^{\infty} S^{\alpha_{i}}$ and $b$ is the compactification point (see [Ch]).

b) if $\left[X_{1}\right]_{X}=X$ and $\left[Y_{1}\right]_{Y}=Y$, then $\left[X_{1} \times Y_{1}\right]_{X \times Y}=X \times Y$.

Let $i: L \hookrightarrow C$ be an embedding of the space $L$ to the Cantor set $C$. Denote $c_{0} L=[i L]_{C}$. Let $M$ be the irrational numbers of the interval $(0,1)$. Observe that $M \simeq L$. Denote $c_{1} L=I=[0,1]$.

It is easy to note that $c_{0} L$ is a zero-dimensional compactification of $L$ and $c_{1} L$ is a one-dimensional compactification of $L$. Let $c_{\alpha} L=c_{\beta} L \times I$ for $\alpha=\beta+1$. If $\alpha$ is a limit ordinal number $<\omega_{1}$, then let $c_{\alpha} L$ be the one-point compactification of the free sum $\bigoplus_{1 \leq \beta<\alpha} c_{\beta} L$ and let $p_{\alpha}$ be the compactification point. It is clear that $c_{\alpha} L$ is a compactification of the space $L$ for any $\alpha<\omega_{1}$.

By induction one can prove the following

Proposition I. For every countable ordinal number $\alpha \geq 1$ we have $S^{\alpha} \hookrightarrow c_{\alpha} L \hookrightarrow S^{\alpha}$.

COROLlary 5. Let $F$ be a monotone dimension function such that

(i) for every ordinal number $\alpha<\omega_{1}$ there exists an ordinal number $\beta<\omega_{1}$ such that $F S^{\beta}=\alpha$;

(ii) $F(X \times Y)=F X$ for any spaces $X, Y$ with ind $Y=0$.

Then for every ordinal number $\alpha<\omega_{1}$ there exists a space $X_{\alpha}$ such that

a) $F X_{\alpha}=\alpha$;

b) for any ordinal number $\beta \geq \alpha$ there exists a compactification $c_{\beta} X_{\alpha}$ with $F c_{\beta} X_{\alpha}=\beta$.

Proof. The spaces $X_{\alpha}$ should be chosen from the collection $\left\{S^{\gamma} \times L: \gamma<\omega_{1}\right\}$ and the compactifications $c_{\beta} X_{\alpha}$ can be found in the collection $\left\{S^{\gamma} \times c_{\beta} L: \gamma, \beta<\omega_{1}\right\}$. Recall ( see [Ch] ) that for any countable ordinal numbers $\nu, \mu$ we have $S^{\nu(+) \mu} \hookrightarrow S^{\nu} \times S^{\mu} \hookrightarrow S^{\nu(+) \mu}$, where (+) is the natural sum of Hessenberg [KM].

REMARK 3. The dimensions trind, D satisfy the conditions of Corollary 5, in particular condition (ii) for trind see [T], for D - [Hel].

Note also that $\operatorname{trInd}\left(S^{\gamma} \times L\right)=\operatorname{trdim}\left(S^{\gamma} \times L\right)=\infty$, if $\gamma \geq \omega_{0}$. 


\section{Questions}

Recall that for every space $X$ with

a) $\operatorname{tr} \operatorname{Ind} X \neq \infty$ there exists a compactification $c X$ such that $\operatorname{trInd} c X=\operatorname{trInd} X$ [Lu1];

b) $\mathrm{D} X \neq \infty$ there exists a compactification $c X$ such that $\mathrm{D} X \leq \mathrm{D} c X \leq \mathrm{D} X+1 \quad[\mathrm{~K}] \quad$ (moreover for every ordinal number $\alpha$ : $\omega_{0} \leq \alpha<\omega_{1}$ there exists a space $X_{\alpha}$ such that $\mathrm{D} X_{\alpha}=\alpha$ and for any compactification $c X_{\alpha}$ of the space $X_{\alpha}$ we have Dc $X_{\alpha}>\alpha$ [Lu1] );

c) $\operatorname{trdim} X \neq \infty$ there exists a compactification $c X$ such that $\operatorname{trdim} c X=\operatorname{trdim} X[\mathrm{Ki}]$.

It is interesting to note that there exists a space $Y$ with $\operatorname{trdim} Y=\omega_{0}+1$ which has a compactification $c Y$ with $\operatorname{trdim} c Y=\omega_{0}$ [B2]. Recall that for dimension trInd, which has very similar properties to dimension trdim, the following statement holds:

if $X \subset Y$ and $\operatorname{trInd} X$, $\operatorname{trInd} Y \neq \infty$, then $\operatorname{trInd} X \leq \operatorname{trInd} Y$ [Lu2].

In connection with this paper one can pose

Problem 1. Let $X$ be a noncompact space, $c X$ be a compactification of the space $X$ and $F(c X) \neq \infty$, where $F$ is one of the functions trind, trInd, D, trdim. Is it true that for any countable ordinal number $\alpha \geq F(c X)$ there exists a compactification $c_{\alpha} X$ such that $F\left(c_{\alpha} X\right)=\alpha$ ?

Let us recall [Lu1] here

LuXEMBURG's CONJECTURE. If $X$ is a space and trind $X=\alpha+p$, where $\alpha$ is a limit ordinal number and $p=0,1,2, \ldots$, then there exists a compactification $c X \supset X$ such that trind $X \leq \alpha+2 p+1$.

\section{REFERENCES}

[AE] J. M. Aarts and P. van Emde Boas, Continua as remainders in compact extensions, Nieuw Arch. Wisk. 15 (1967), 34-37.

[AP] P. S. Aleksandrov and B. A. Pasynkov, Introduction to Dimension Theory (Russian), Moscow 1973.

[B1] P. Borst, Transfinite Classifications of Weakly Infinite-dimensional Spaces, dissertation, Amsterdam 1986.

[B2] P. Borst, On weakly infinite-dimensional subspaces, Fund.Math. 140 (1992), 225-235.

[Ch] V. A. Chatyrko, Ordinal products of topological spaces, Fund. Math. 144 (1994), 95-117.

[E] R. Engelking, Theory of Dimensions Finite and Infinite, Sigma Ser. Pure Math. vol. 10, 1995.

[Ha] Y. Hattori, Remarks on weak large transfinite dimension w-Ind, Q \& A in General Topology 4 (1986), 59-66. 
[He1] D. W. Henderson, D-dimension, I. Anew transfinite dimension, Pacific J. Math. 26 (1968), 91-107.

[He2] D. W. Henderson, A lower bound for transfinite dimension, Fund. Math. 64 (1968), 167173.

[Ki] T. Kimura, A note on compactification theorem for trdim, Topology Proc. 20 (1995), 145159.

[K] I. M. Kozlovski, Two theorems on metric spaces, Soviet Math. Dokl. 13 (1972), 743-747.

[KM] K. Kuratowski, A. Mostowski, Set Theory, PWN, 1976.

[Lu1] L. A. Luxemburg, On compactifications of metric spaces with transfinite dimensions, Pacific. J. Math. 101 (1982), 399-450.

[Lu2] L .A. Luxemburg, On transfinite inductive dimensions, Soviet Math. Dokl. 14 (1973), 388-393.

[Le] B. T. Levshenko, Spaces of transfinite dimensionality, Amer. Math. Soc. Transl. 73 (1968), 135-148.

[S] Ju. M. Smirnov, On universal spaces for certain classes of infinite-dimensional spaces, Amer. Math. Soc. Transl. 21 (1961), 35-50.

[T] G. H. Toulmin, Shuffling ordinals and transfinite dimension, Proc. London Math. Soc. 4 (1954), 177-195.

DEPARTMENT OF MATHEMATICS

LINKÖPING UNIVERSITY

58183 LINKÖPING

SWEDEN

Email: vitja@mai.liu.se 